\section{Sarcoidosis secondary to lymphocyte active immunotherapy treated with infliximab*}

Gabriela Higino de Souza ${ }^{1}$

Chan I Thien ${ }^{1}$

Victor Bechara de Castro ${ }^{1}$

Alexandre Carlos Gripp ${ }^{1}$

DOI: http:/ / dx.doi.org/10.1590/abd1806-4841.20187624

Dear Editor,

We report a case of a 38-year-old woman who presented a pustule with erythematous base in the right deltoid region after application of lymphocyte active immunotherapy (LAI) to treat infertility (reported history of an abortion). Subsequently, a similar, exudative lesion appeared in the left deltoid region. New lesions appeared bilaterally, forming hardened erythematoviolaceous plaque (Figure 1). The same treatment, applied on both arms, was administered four years prior without complications. Given the clinical picture, the diagnostic hypothesis of sarcoidosis was suggested. A biopsy was performed, showing epithelioid cell granulomas without lymphocyte border ("naked granuloma") and multinucleated histiocytes (giant cells) in the dermis, compatible with sarcoidosis (Figure 2). The patient presented no respiratory symptoms; however, the computed tomography (CT) of her thorax showed intrathoracic and bilateral hilar lymphadenopathy. Infiltration of intralesional triamcinolone, hydroxychloroquine and subcutaneous methotrexate $(25 \mathrm{mg} /$ week) obtained no response. Treatment with infliximab (5mg/ $\mathrm{kg} /$ infusion) was then initiated, with induction at weeks 0 , 2 , and 6 , followed by infusions every eight weeks for maintenance. Thus far, induction has been realized, and two maintenance doses have been administered (weeks 14 and 22), with significant clinical

\footnotetext{
Received 10 September 2017.

Accepted 17 February 2018.

* Work conducted at the Clinic of Immunobiologicals in Dermatology, Hospital Universitário Pedro Ernesto, Universidade do Estado do Rio de Janeiro, Rio de Janeiro (RJ), Brazil.

Financial support: None.

Conflict of interest: None.

1 Immunobiology clinic, Hospital Universitário Pedro Ernesto, Universidade do Estado do Rio de Janeiro, Rio de Janeiro (RJ), Brazil.

MaILING AdDRESS:

Victor Bechara de Castro

E-mail: becharavic@yahoo.com.br

@ 2018 by Anais Brasileiros de Dermatologia

(cc) BY-NC
}

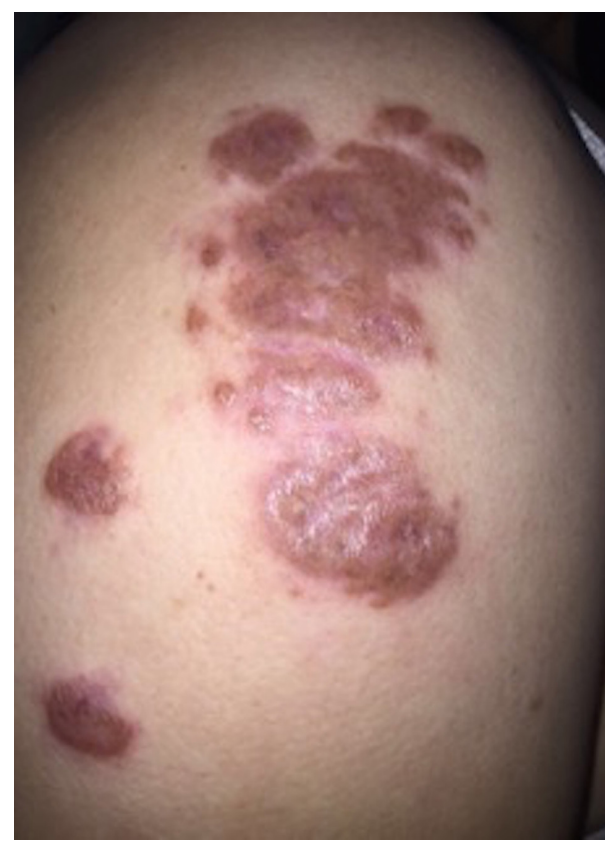

Figure 1: Erythematoviolaceous, infiltrated, hardened plaques located in the right deltoid region, before initiation of treatment

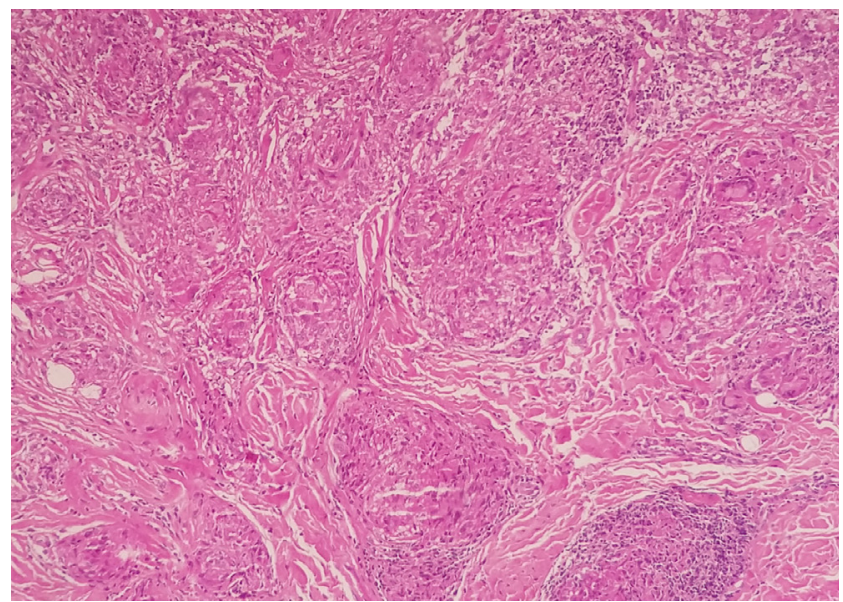

FIGURE 2: Histopathological exam showing epithelioid cell granulomas without lymphocyte border ("naked granuloma"), surrounded by fibroplasia (Hematoxylin \& eosin, x100)

improvement at week 6 of induction. Residual cutaneous lesions were noted at week 14 of treatment, and a new CT of the thorax did not detect hilar lymphadenopathy (Figure 3). The patient continued with periodic follow-up, using maintenance dosing of infliximab every eight weeks, without relapse of the lesions.

Sarcoidosis is an immune-mediated inflammatory disease characterized by the formation of non-caseating granulomas. It can affect different organs, and cutaneous involvement varies from small lesions to extensive presentations, with impacts on quality of life. ${ }^{1}$ The diagnosis is made according to clinical, radiological and 


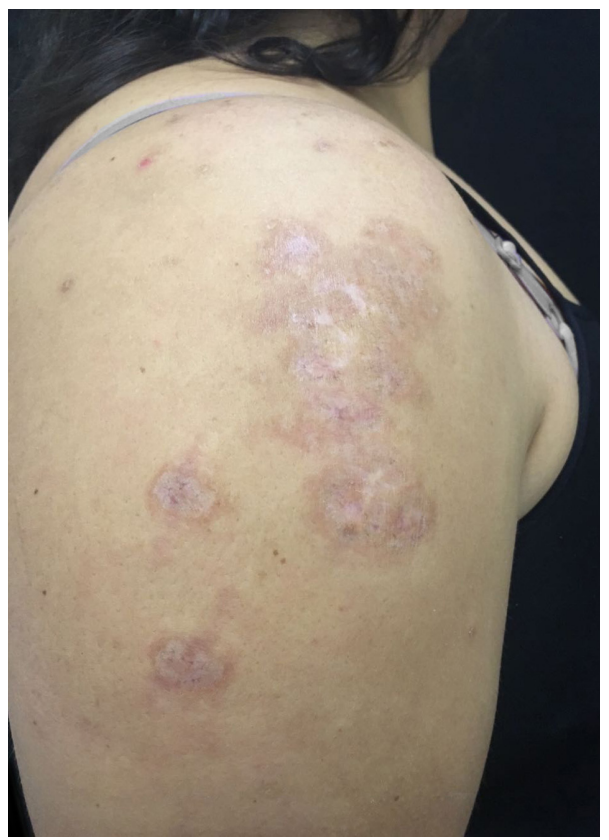

Figure 3: Brownish erythematous, atrophic and residual plaques in the right deltoid region after induction (weeks 0, 2 and 6) and two maintenance doses (weeks 14 and 22) of infliximab histopathological data, after exclusion of differential diagnoses. ${ }^{2}$ Although the cause is yet unknown, ${ }^{1,2}$ numerous works have proposed to demonstrate substances that may function as triggering or aggravating factors or may simply be related to its physiopathogeny. It is believed that a certain agent, infectious or not, must act on an immunogenetically predisposed individual. ${ }^{2}$ In the formation of sarcoidal granuloma, innumerable cells, interleukins, and intercellular communication pathways are involved. Among them, the lymphocytes T CD4+ are highlighted. ${ }^{1,2}$ Interleukin-2, interferon gamma, and tissue necrosis factor-alpha play important roles. ${ }^{1,2}$

The disease can appear on areas of damaged skin, such as tattoos, and at previous intramuscular or intravenous injection sites, for example. ${ }^{1}$ Likewise, there are described cases in which the disease developed after a vaccination. ${ }^{3}$ In the reported case, the injected material was a lymphocyte concentration applied intradermally. LAI is considered a therapeutic option for patients with recurrent miscarriages and uses lymphocytes obtained from the patient's male partner or from a healthy donor. The objective is to produce blocking antibodies, which act against the rejection of the embryo to reduce the risk of miscarriage. ${ }^{4}$ According to the literature, after the exclusion of hepatitis B and C, syphilis and HIV infection, venous blood of the donor is collected and treated with an anticoagulant (heparin). The lymphocytes are separated, washed and diluted in saline solution. The injections are applied at four to six sites on the patient's upper limb and repeated at 2- or 3-week intervals. However, the therapeutic scheme and its efficacy remain controversial. ${ }^{4}$ It is believed that the immunotherapy functions as an antigen, with possible activation of reactive $\mathrm{T}$ cells, stimulating the onset of an inflammatory reaction of the damaged skin, and that this reaction is sufficiently intense to persist until the full development of the disease.
The treatment of sarcoidosis is not curative, and it alters the granulomatous process and its clinical consequences. ${ }^{2}$ There is no strict consensus. The use of tetracyclines or hydroxychloroquine is described, though with a low level of evidence of their efficacy. The use of systemic corticosteroid, methotrexate and thalidomide is an option for extensive and refractory cases. ${ }^{1,2}$ Studies on the use of anti-TNF-alpha agents are scarce. Heidelberger et al., ${ }^{5}$ in an observational multicentric study of 46 patients with cutaneous sarcoidosis, observed good response to the use of infliximab: significant response in $24 \%$ after three months; $46 \%$ after six months; and $79 \%$ after twelve months. However, attention must be given to the possibility of recurrence after suspending medication and to the risk of adverse effects, such as infections.

The presentation of this case is justified by the rarity of sarcoidosis induced by the LAI. The association is currently uncommon; however, new cases may arise as infertile couples seek this therapeutic option. More studies are needed to clarify the physiopathogenesis of sarcoidosis resulting from immunotherapy. In addition to discontinuation of the suspected inducing agent, treatment of this clinical presentation can be achieved with infliximab, showing satisfactory response. Attention should be given to possible adverse events and to relapse after suspension of treatment. $\square$

\section{REFERENCES}

1. Wanat KA, Rosenbach M. Cutaneous Sarcoidosis. Clin Chest Med. 2015;36:685702.

2. Valeyre D, Prasse A, Nunes H, Uzunhan Y, Brillet PY, Müller-Quernheim J. Sarcoidosis. Lancet. 2014;383:1155-67.

3. Osborne GE, Mallon E, Mayou SC. Juvenile sarcoidosis after BCG vaccination. J Am Acad Dermatol. 2003;48:S99-102.

4. Chen JL, Yang JM, Huang YZ, Li Y. Clinical observation of lymphocyte active immunotherapy in 380 patients with unexplained recurrent spontaneous abortion. Int Immunopharmacol. 2016;40:347-50.

5. Heidelberger V, Ingen-Housz-Oro S, Marquet A, Mahevas M, Bessis D, Bouillet L, et al. Efficacy and Tolerance of Anti-Tumor Necrosis Factor $\alpha$ Agents in Cutaneous Sarcoidosis: A French Study of 46 Cases. JAMA Dermatol. 2017;153:681-5.

AUTHORS CONTRIBUTIONS
Gabriela Higino de Souza
Approval of the final version of the manuscript, Preparation and writing of the manu-
script, Collecting, analysis and interpretation of data, Intellectual participation in pro-
paedeutic and/or therapeutic conduct of studied cases, Critical review of the literature,
Critical review of the manuscript
Chan I Thien
Approval of the final version of the manuscript, Preparation and writing of the manu-
script, Effective participation in research orientation, Intellectual participation in pro-
paedeutic and/or therapeutic conduct of studied cases, Critical review of the literature,
Critical review of the manuscript
Victor Bechara de Castro
Statistical analysis, Approval of the final version of the manuscript, Preparation and
writing of the manuscript, Critical review of the manuscript
Alexandre Carlos Gripp
Approval of the final version of the manuscript, Critical review of the literature, Critical
review of the manuscript

How to cite this article: Souza GH, Chan IT, Castro VB, Gripp AC. Sarcoidosis secondary to lymphocyte active immunotherapy treated with infliximab. An Bras Dermatol. 2018;93(5):764-5. 